\title{
DOE-EPSCOR SPONSORED PROJECT FINAL REPORT
}

DOE Award Number: DE-FG02-06ER46265

Recipient Organization: Desert Research Institute, Division of Hydrologic Sciences, 2215 Raggio Parkway, Reno, Nevada 89512-1095, and 755 E Flamingo Road, Las Vegas, Nevada 89119

Project Title: A New Method to Estimate Soil Hydraulic Parameter Uncertainty and Heterogeneity Using Bayesian Updating and an Artificial Neural Network

Principal Investigator: Jianting Zhu (Jianting.Zhu@dri.edu, Tel: 702-862-5416, Fax: 702862-5427)

Date of the Award Period: January 1, 2006 to December 31, 2009 (three-year project from January 1, 2006 to December 31, 2008 with one-year no cost extension from January 1, 2009 to December 31, 2009)

Total Approved Budget: $\$ 357,898$ from DOE, $\$ 35,790$ match from Applied Research Initiative of Nevada

Year 1 budget: \$104,466 from DOE, \$10,447 match from Applied Research Initiative of Nevada

Year 2 budget: $\$ 120,424$ from DOE, \$12,042 match from Applied Research Initiative of Nevada

Year 3 budget: \$133,008 from DOE, \$13,301 match from Applied Research Initiative of Nevada

National Laboratory Collaborator: Pacific Northwest National Laboratory

Sponsor: DOE EPSCoR

Submitted to EPSCoR Program, U. S. Department of Energy

March, 2010 


\section{DESCRIPTION OF ACCOMPLISHMENTS}

\section{Introduction and Objectives}

Concern over the quality of environmental management and restoration has motivated the model development for predicting water and solute transport in the vadose zone. Soil hydraulic properties are required inputs to subsurface models of water flow and contaminant transport in the vadose zone. Computer models are now routinely used in research and management to predict the movement of water and solutes into and through the vadose zone of soils. Such models can be used successfully only if reliable estimates of the soil hydraulic properties are available. The hydraulic parameters considered in this project consist of the saturated hydraulic conductivity, $K s$, and four parameters of the water retention equation by the van Genuchten equation,

$$
\theta(\psi)=\theta_{r}+\frac{\left(\theta_{s}-\theta_{r}\right)}{\left[1+(\alpha \psi)^{n}\right]^{m}}
$$

where $\psi$ is the capillary pressure head, $\theta$ is the soil water content, $\theta_{r}$ is the residual water content of soil, $\theta_{s}$ is the saturated water content of soil, $\alpha$ is the shape factor, $n$ is the pore size distribution index, and $m$ is the empirical constant which can be related to $n$ by $m=$ $1-1 / n$.

The overall objective of this project is to better quantify soil hydraulic parameters which are critical in predicting water flows and contaminant transport in the vadose zone through a comprehensive and quantitative study to predict heterogeneous soil hydraulic properties and the associated uncertainties. We seek to address a number of important issues related to the soil hydraulic property characterizations. The results of numerical simulations of a field injection experiment at Hanford site in this project can be used to provide insights to the DOE mission of appropriate contamination characterization and environmental remediation. This project also strengthens collaboration between researchers at the Desert Research Institute in the EPSCoR State of Nevada and their colleagues at the Pacific Northwest National Laboratory. While all of our efforts of this project are centered on estimating heterogeneous soil hydraulic parameters, evaluating the associated uncertainty and applying to field applications at the Hanford site, and innovative approaches of quantifying soil hydraulic properties applicable to large scale modeling of hydrological processes including flow and contaminant transport in the vadose zone, we report in the following topics roughly in the chronological order when the work was performed or published for the organization of the report. All the reported work has been published, under peer review or is under preparation to be submitted to peer-reviewed journals in the near future.

\section{Macroscopic Parallel and Perpendicular Unsaturated Hydraulic Conductivities for Layered Soils}

It is a well known fact that for saturated flow the macroscopic scale hydraulic conductivity is equal to a simple arithmetic average of all individual saturated hydraulic conductivities for horizontally heterogeneous media and harmonic average of all individual saturated hydraulic conductivity for vertically heterogeneous media. The same average schemes were often assumed and extended to the more complex unsaturated flows in many studies. Due to the strongly non-linear relationship between the unsaturated hydraulic conductivity and the saturation degree, the validity of the same 
average schemes for unsaturated flow conditions needs to be examined. We investigated the appropriateness of using the arithmetic and harmonic averages of hydraulic conductivity for unsaturated flows horizontal and perpendicular to the heterogeneous soil columns or layers under the steady state flow conditions (Zhu, 2008). The macroscopic hydraulic conductivity in this study meant the derived equivalent macroscopic hydraulic conductivity from either horizontally or vertically heterogeneous soil columns or layers distinguishable by variations in the hydraulic parameters. Specifically, for horizontally heterogeneous soils the macroscopic hydraulic conductivity function should be able to predict the actual average flux of the parallel column soil system and for vertically heterogeneous soils, it should simulate the actual moisture flux of the layered soil formations.
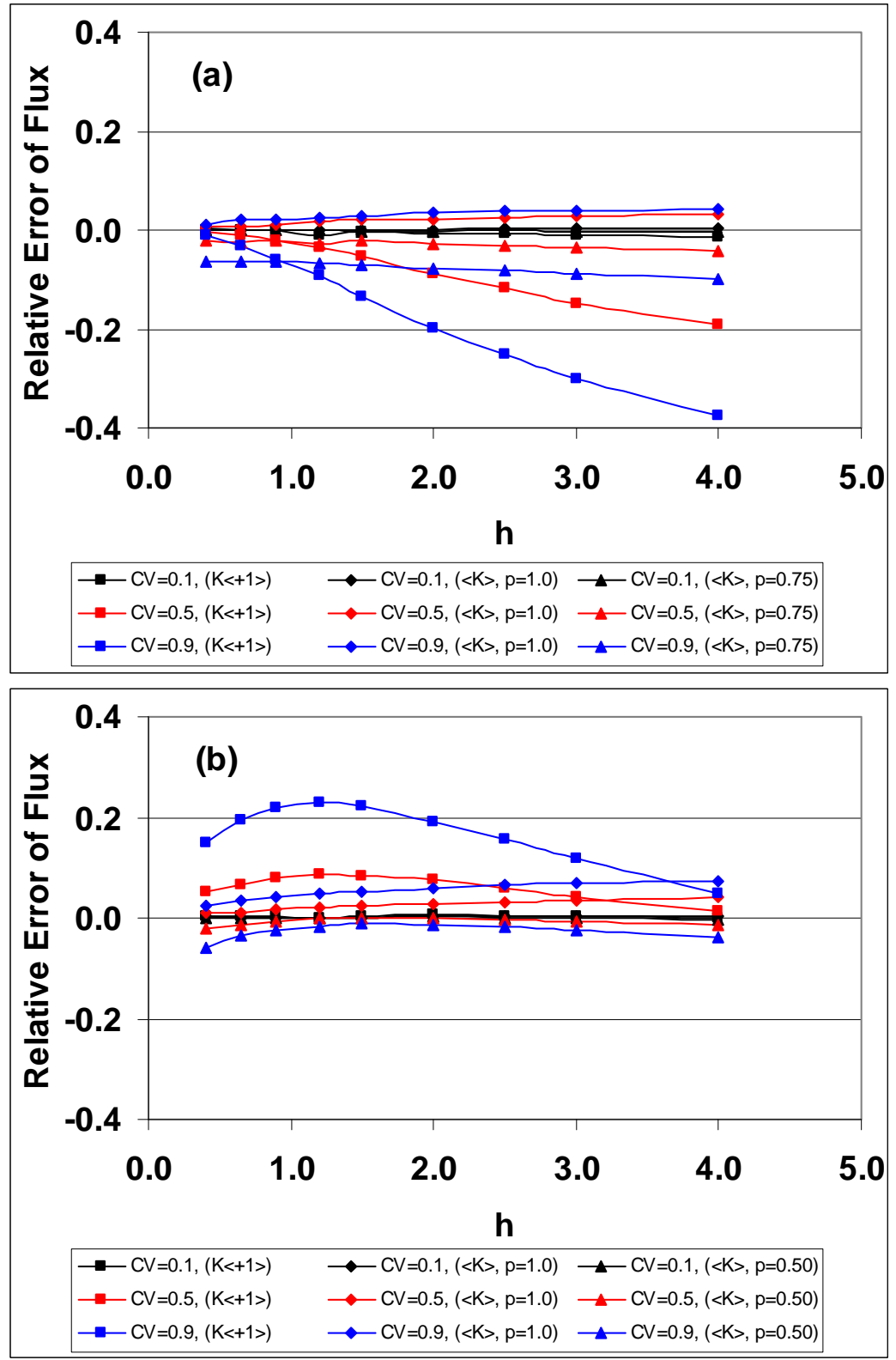

Figure 1: Relative error of flux as functions of $h$ under various average hydraulic conductivity schemes for horizontal heterogeneity scenario for $\left\langle\alpha^{*}\right\rangle=1$ at selected variance levels $\left(\mathrm{CV}=C V_{\alpha^{*}}\right.$ $=C V_{K s}$ values of $0.1,0.5$, and 0.9$)$. (a) $\mathrm{r}=0.0$, (b) $\mathrm{r}=0.9$. 

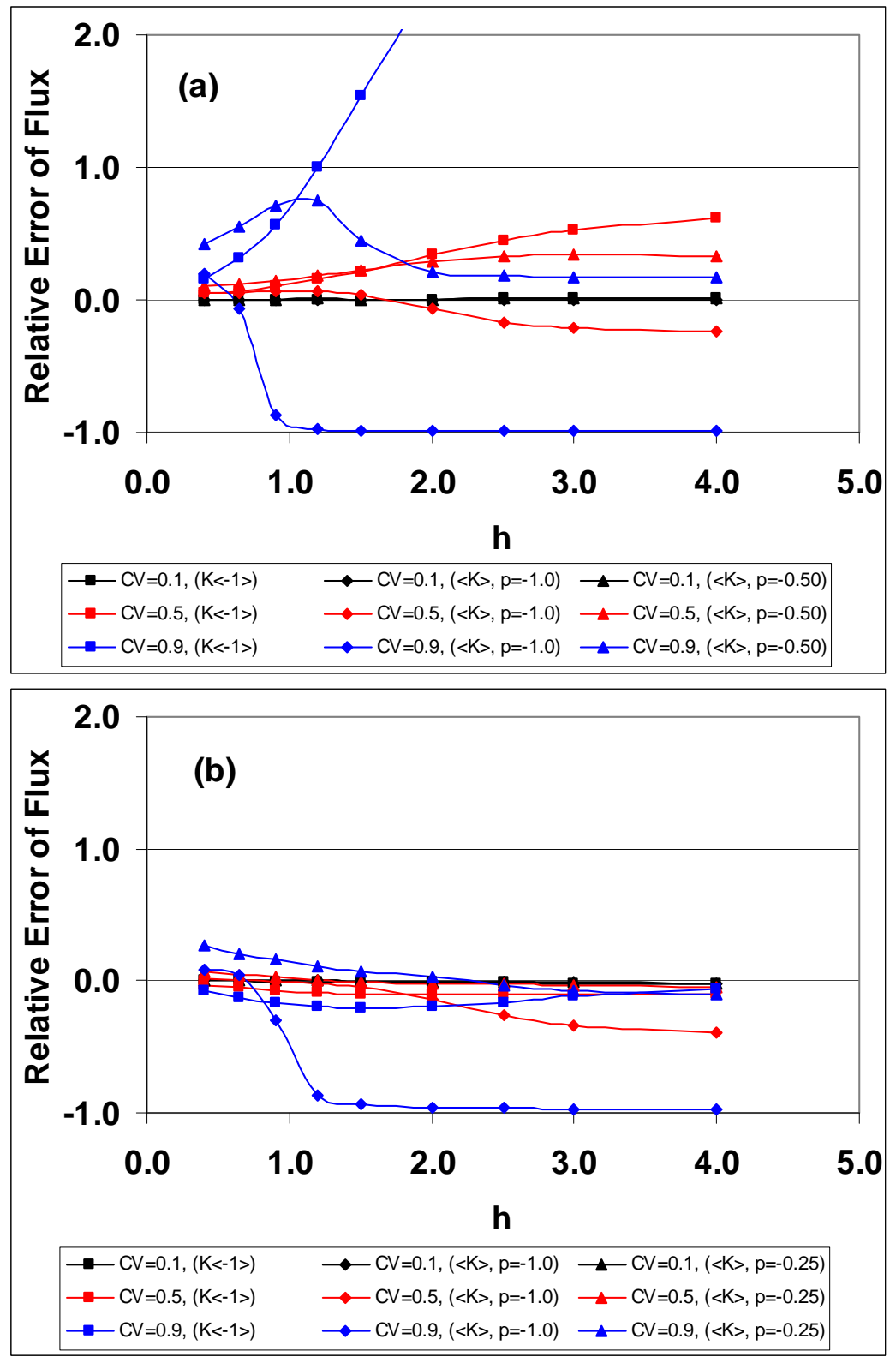

Figure 2: Relative error of flux as functions of $h$ under various average hydraulic conductivity schemes for vertical heterogeneity scenario for $\left\langle\alpha^{*}\right\rangle=1$ at selected variance levels $\left(\mathrm{CV}=C V \alpha^{*}=\right.$ $C V_{K s}$ values of $0.1,0.5$, and 0.9 ). (a) $r=0.0$, (b) $r=0.9$.

The relative error of calculated flux against the actual average flux of the heterogeneous soils, using various averaged hydraulic conductivity functions was also assessed. Figure 1 and Figure 2 illustrated errors Relative error of flux as functions of the dimensionless ground surface capillary pressure head $(\mathrm{h})$ under various average hydraulic conductivity schemes for horizontal heterogeneity scenario and vertical heterogeneity scenario respectively. In general, our results illustrated that while arithmetic mean of hydraulic conductivity function performed reasonable well in predicting the actual average flux for the horizontally heterogeneous columns, the harmonic mean of hydraulic conductivity function introduced quite large error in predicting the actual flux for the 
vertically heterogeneous soil layers, especially for the highly heterogeneous soils. Some of the other main conclusions from this study were: (1) the optimal macroscopic hydraulic conductivities for both horizontally and vertically heterogeneous soils also depended on the capillary pressure head conditions at the land surface, and it was more challenging when the capillary pressure head at the land surface was higher (i.e., dry surface conditions); (2) the arithmetic mean for horizontally heterogeneous soil columns and harmonic mean for vertically heterogeneous soil layers, extended from saturated flow situations, would introduce larger errors in simulating the actual flux for coarser textured soils and for more heterogeneous soils with large hydraulic parameter variances; and (3) the parametric correlations among the hydraulic parameters were also important in determining the appropriate macroscopic hydraulic conductivity for the heterogeneous soils.

\section{Estimating Heterogeneous Hydraulic Properties using Cokriging and Artificial Neural Network and Simulation of Field Injection Experiment at Hanford Site}

We developed an approach to integrate data that are easily available (for example, initial moisture content, bulk density, and soil texture) with soil hydraulic property data via cokriging and artificial neural network (ANN)-based pedotransfer functions (PTFs) (Ye et al., 2007). The method was applied to generate heterogeneous soil hydraulic parameters at a field injection site at the Hanford area. Cokriging was first used to generate three-dimensional heterogeneous fields of bulk density and soil texture using an extensive data set of field-measured moisture content, which carry signature about site heterogeneity and stratigraphy. Soil texture and bulk density were subsequently input into an ANN-based site-specific PTF to generate three-dimensional heterogeneous soil hydraulic parameter fields. The stratigraphy at the site was well represented by the estimated pedotransfer variables and the soil hydraulic parameters. The soil hydraulic property data included laboratory measurements of saturated hydraulic conductivity and van Genuchten moisture retention parameters obtained from 70 core samples at the Hanford area. Another type of data used, also referred to as pedotransfer variables, included bulk density and percentages of gravel, coarse sand, fine sand, silt, and clay measured for the same 70 core samples. The data set also included 1,376 observations of initial moisture content before the injection experiment at the Hanford area. The hydraulic parameter estimates were then used to simulate the field injection experiment at the Hanford 200E Area. Figure 3 showed the comparison of first and second spatial moments of soil moisture evolution between the observed and simulated results. A relatively good agreement was achieved between the simulated and observed moisture contents. The spatial distribution pattern of observed moisture content as well as the moisture movement was captured reasonably well in the simulations. In contrast to earlier work using an effective parameter approach, we were able to reproduce the observed moisture plume in a coarse sand unit that was sandwiched between two fine-textured units. The approach of combining cokriging and ANN for site characterization of heterogeneous soils provided unbiased prediction of the observed moisture plume and was flexible so that additional measurements of various types can be included as they become available. 

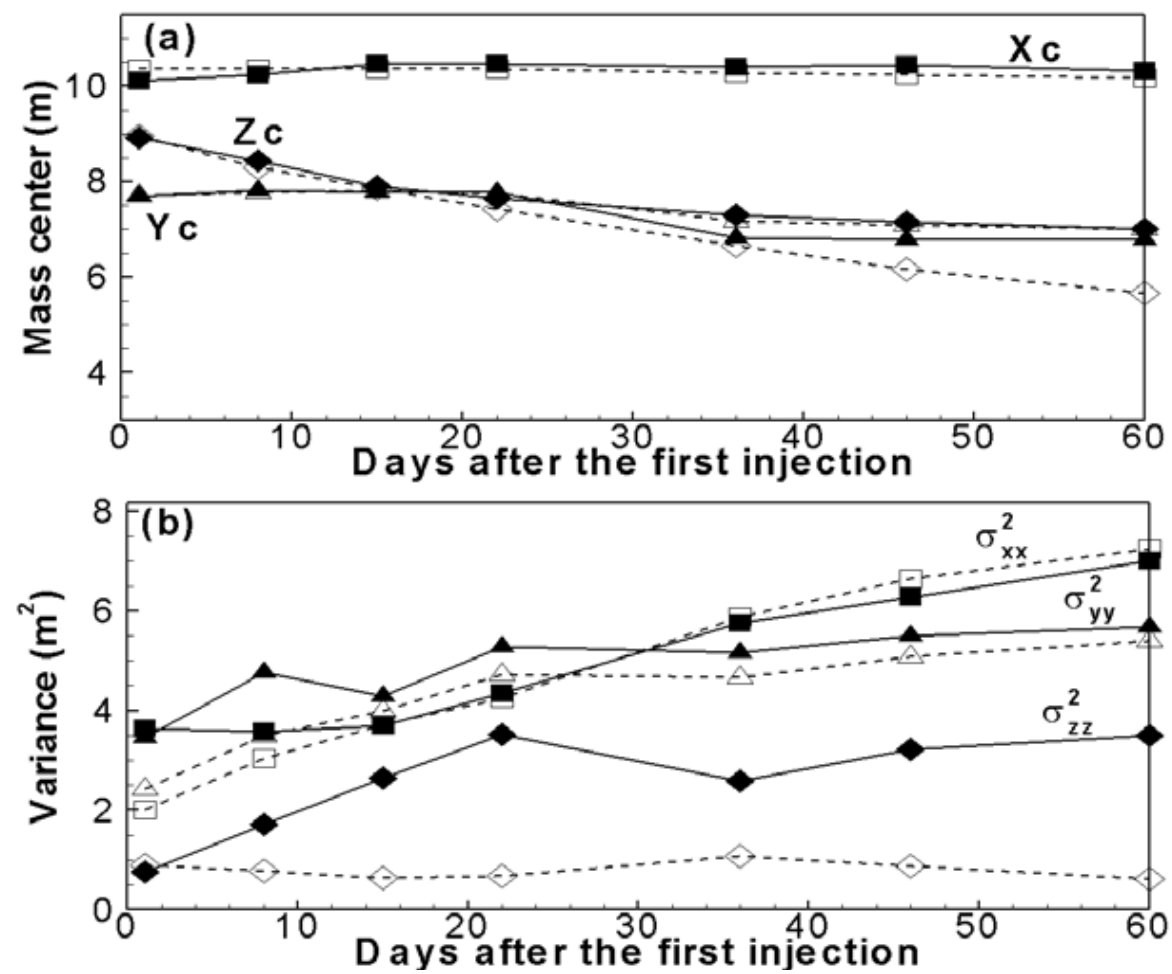

Figure 3: Comparison of observed (filled symbol and solid line) and simulated (open symbol and dashed line) (a) first and (b) second moment of moisture plume

\section{Markov Chain Model for Characterizing Medium Heterogeneity and Sediment Layering Structure}

By leveraging use of "soft" data (e.g., initial moisture content), the work of Ye and Khaleel (2008) applied the transition probability (TP) based Markov chain (MC) model to sediment textural classes for characterizing the medium heterogeneity and sediment layering structure. The TP/MC method was evaluated by simulating the vadose zone moisture movement at the field injection site at Hanford, where the stratigraphy consists of imperfectly stratified soil layers. Soil heterogeneity was characterized via spatial variability of the geometry of soil textural classes. When the initial moisture content measurements, which carry signature about medium heterogeneity and stratigraphy, were not included in the TP/MC model, it was not possible to identify the horizontal TP. The initial moisture content measurements, when transformed into soil classes, were necessary in mapping the soil layering structure prevalent at the site. The soil hydraulic parameters for each soil class were treated deterministically and were estimated on the basis of core samples. To evaluate uncertainty in characterizing geometry of the soil classes, multiple conditional realizations of the soil classes were generated. A Monte Carlo simulation showed that the simulated mean moisture contents agree well with corresponding field observations. Figure 4 showed comparison between the threedimensional contours of observed and simulated mean moisture content on several dates. Pearson's linear correlation coefficients (r) were calculated for the two simulation dates. The observed splitting of the moisture plume in a coarse sand layer that is sandwiched between two fine-textured layers, the southeastward movement of the plume during the redistribution period, and the near-zero fluid flux below the bottom fine layer were 
adequately simulated. Spatial variability of the field-measured moisture content was sufficiently captured by the $95 \%$ confidence intervals calculated from the Monte Carlo simulations. Investigating the effect of data conditioning on the simulated results showed that a reduction of conditioning data does not necessarily deteriorate simulation results if other conditioning data exist within the mean length of the soil classes. The TP/MC method was flexible so that other types of site characterization data (e.g., geophysical data) can be incorporated as they become available.

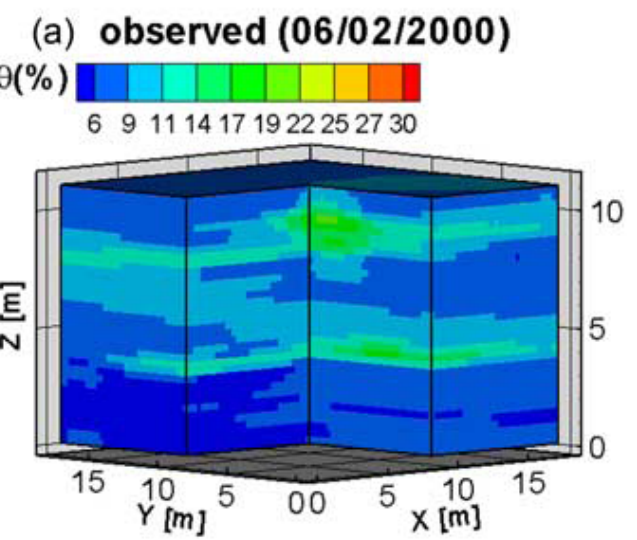

(c) simulated $(06 / 02 / 2000)$

$\theta(\%)$

6991114171922252730
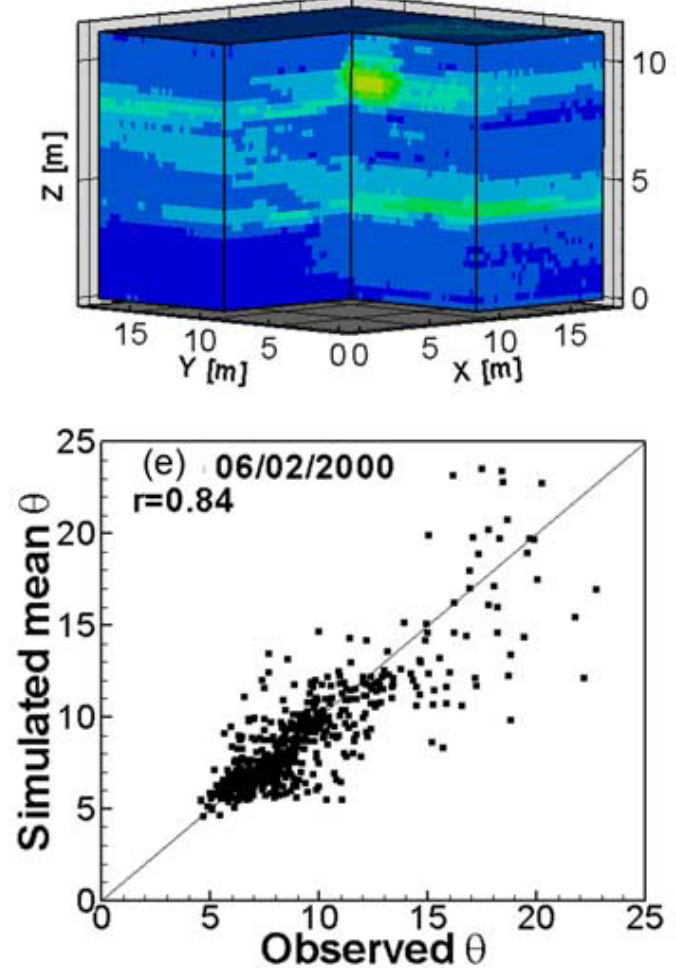

(b) observed $(07 / 31 / 2000)$

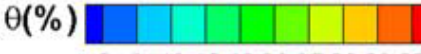

691216192225293235

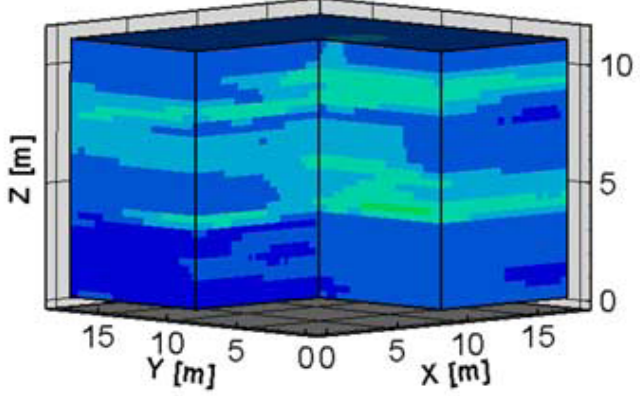

(d) simulated $(07 / 31 / 2000)$

$\theta(\%)$

691216192225293235
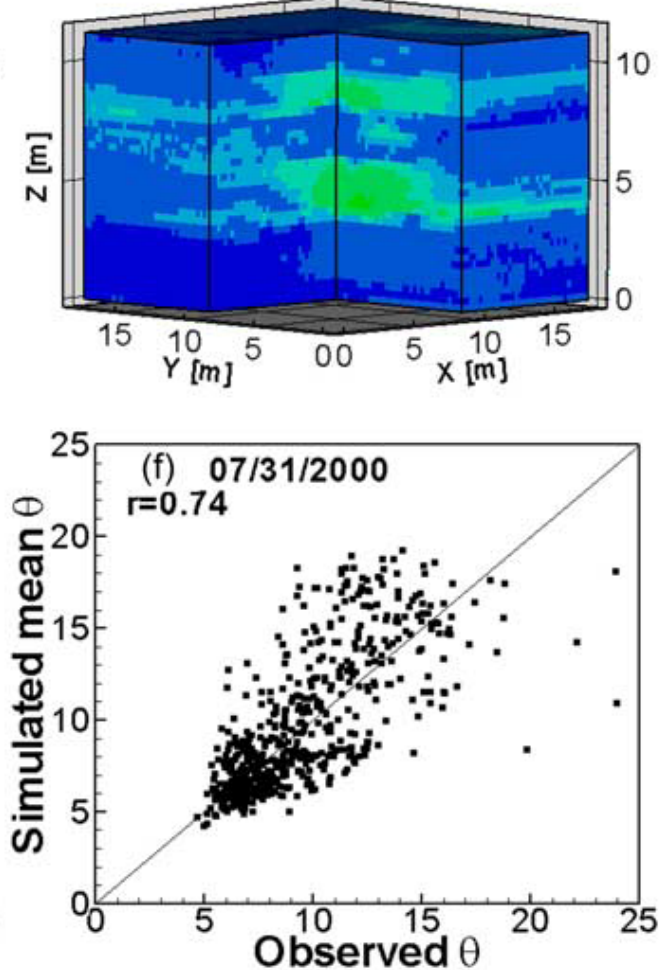

Figure 4: Three-dimensional contours of (a) observed and (c) simulated mean moisture content on 2 June 2000; three-dimensional contours of (b) observed and (d) simulated mean moisture content on 31 July 2000; and observed and simulated mean water content on (e) 2 June 2000 and (f) 31

July 2000. Pearson's linear correlation coefficients (r) were calculated for the two simulation times 


\section{Effective Soil Hydraulic Parameters for Transient Flows in Heterogeneous Soils}

The study of Zhu and Sun (2009) investigated the use of effective soil hydraulic properties (expressed in terms of hydraulic parameters) applicable to large-scale transient infiltration problems in a landscape with horizontally heterogeneous soil hydraulic properties. The heterogeneous landscape was conceptualized as an equivalent homogeneous medium with effective hydraulic properties. The main objectives were to investigate: (i) which effective soil hydraulic property schemes are suitable to represent average behavior of large-scale infiltration processes, (ii) how the effective hydraulic parameters are sensitive to the process time frame, and (iii) how hydraulic parameter variability and correlation impact the effective hydraulic parameters. The heterogeneous landscape was represented by a series of vertically homogeneous stream tubes or parallel columns. Large-scale average infiltration behavior in the heterogeneous soils was quantified through Monte Carlo simulations of multiple realizations (stream tubes) of local-scale infiltration. The optimal effective hydraulic parameters were then calculated with an inverse procedure that minimized the difference between average cumulative infiltration and cumulative infiltration based on a single set of effective parameters.
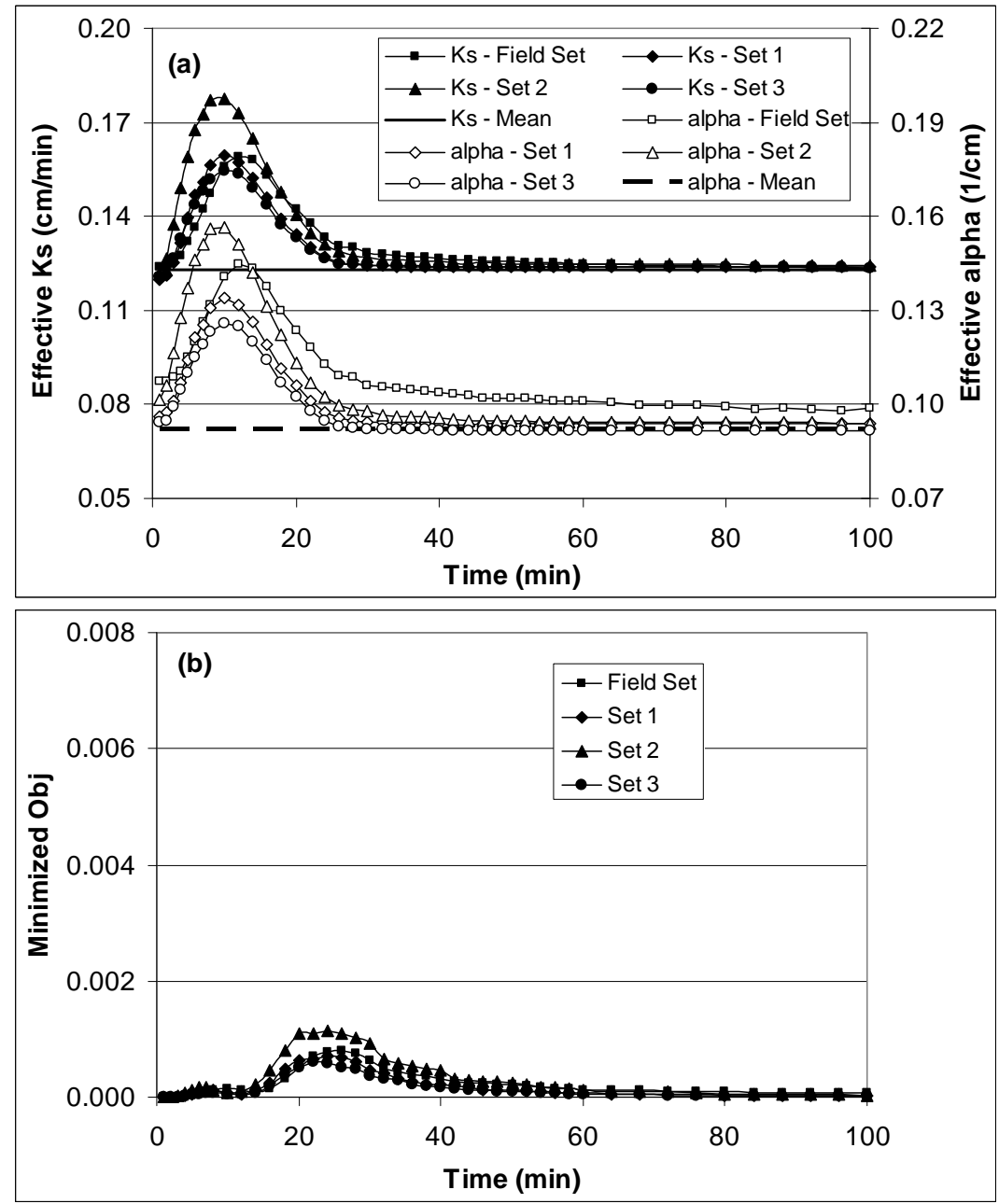

Figure 5: Effective hydraulic parameters and corresponding minimized objective functions for cumulative infiltration as a function of time when both saturated hydraulic conductivity $\mathrm{K}_{\mathrm{S}}$ and

shape factor $\alpha$ are optimized simultaneously. (a) Effective hydraulic parameters, and (b) minimized objective functions 
Three scenarios were used to optimize either the hydraulic parameters simultaneously or only one hydraulic parameter while using the arithmetic mean for the other parameter. Several hydraulic parameters data sets were synthetically generated in order to investigate the impact of different statistics of hydraulic parameters on the effective hydraulic parameter schemes. Figure 5 showed effective hydraulic parameters and corresponding minimized objective functions for cumulative infiltration as a function of time. Results indicated that while the effective hydraulic parameters could simulate average infiltration more closely when multiple parameters were optimized together, the effective parameter values were more variable as time evolved. Optimizing only one hydraulic parameter while keeping the arithmetic mean for the other parameter produced more uniform effective hydraulic parameters with time, but this approach did not represent average infiltration behavior of the heterogeneous soils as well as when multiple hydraulic parameters were optimized simultaneously.

Note: The progresses reported in 5 through 9 below were made largely from the third year the no cost extension fourth year although much work actually started during the first two years.

\section{Quantification of Uncertainty in Pedotransfer Function-Based Parameter Estimation for Unsaturated Flow Modeling}

The objective of the study by Deng et al. (2009) was to evaluate uncertainty of the PTF-estimated soil hydraulic parameters and its effect on numerical simulation of moisture flow. Contributing to the parameter estimation uncertainty are (1) the PTF intrinsic uncertainty caused by limited data used for PTF training and (2) the PTF input uncertainty in pedotransfer variables (i.e., PTF inputs). The PTF intrinsic uncertainty was assessed using the bootstrap method by generating multiple bootstrap realizations of the soil hydraulic parameters; the realizations followed normal or lognormal distributions. The PTF input variables (i.e., bulk density and soil texture) were obtained using the cokriging technique. The PTF input uncertainty was quantified by assuming that the cokriging estimates follow a normal distribution. Figure 6 showed comparison of the observed and simulated moisture contents on 23 June 2000 at four boreholes. The results showed that the PTF input uncertainty dominates over the PTF intrinsic uncertainty and determines the spatial distribution of the PTF parameter estimation uncertainty. When the parameter estimation uncertainty was included, the spatial variability of the measured soil hydraulic parameters was better captured. This was also the case for the observed moisture contents, whose spatial variability was well bracketed by the prediction intervals. However, this was only possible after the PTF input uncertainty was considered. These results suggested that additional sample acquisition for the PTF input variables (soil texture and bulk density) would have a more favorable impact on reduction of the parameter estimation uncertainty than collecting additional soil hydraulic parameter measurements for PTF development. Therefore, it appeared that a more complete characterization of a site for texture and layering and a judicious positioning of sample measurement locations would lead to an optimal site characterization of soil hydraulic parameter heterogeneity. 

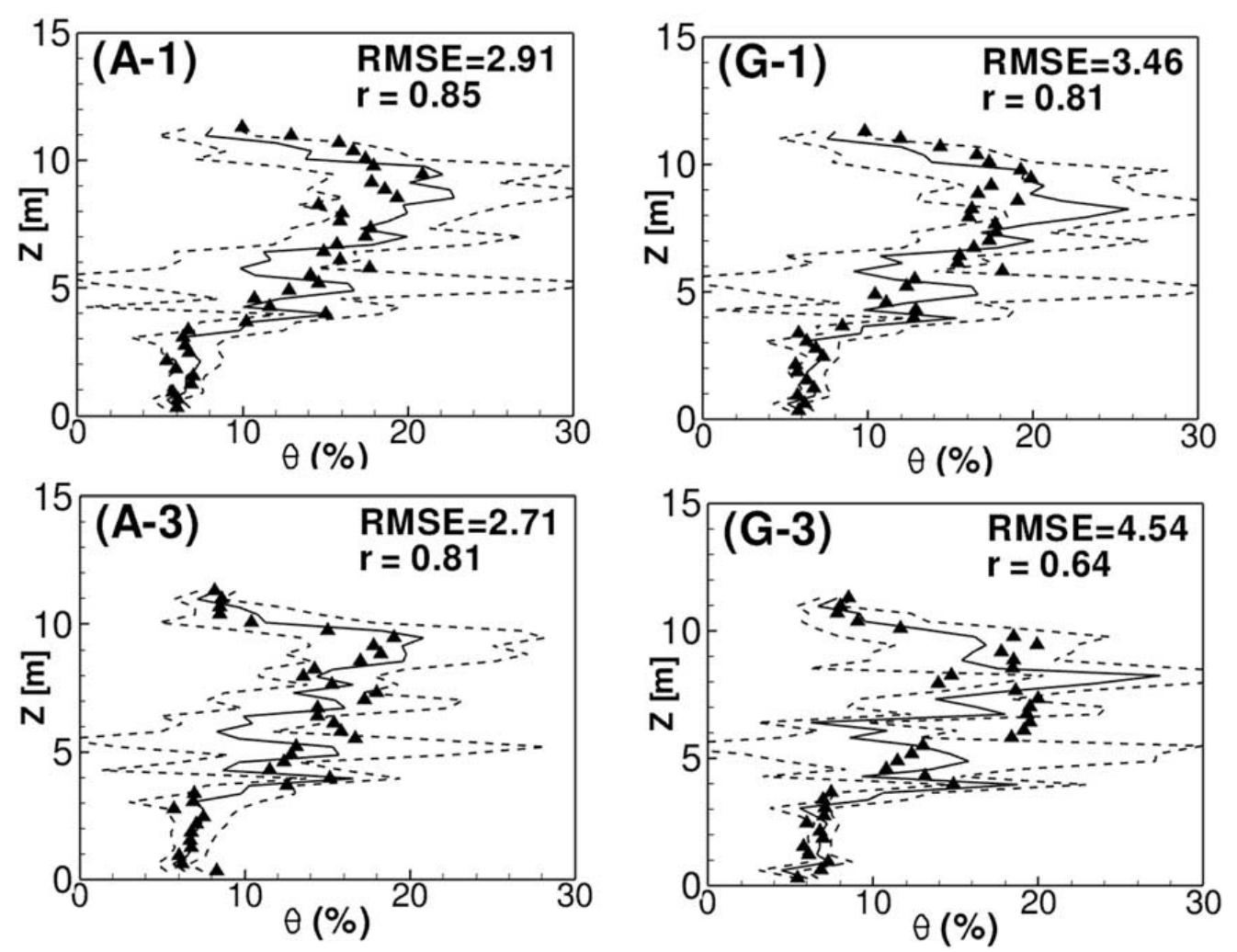

Figure 6: Comparison of the observed (triangles) and simulated moisture contents on 23 June 2000 at four boreholes. The mean and $95 \%$ prediction intervals of the simulated moisture content are shown as solid and dashed lines, respectively. Both PTF intrinsic and input uncertainty are considered

\section{Unsaturated Hydraulic Conductivity for Layered Soils of Structured Heterogeneity}

The study of Zhu and Warrick (2009) investigated hydraulic conductivities of unsaturated soils for one-dimensional structured heterogeneity. The heterogeneity was defined by forming repeated unit cells of homogeneous sublayers. Specifically, we examined the appropriateness of harmonic mean of unsaturated hydraulic conductivities in representing the structured heterogeneity for a finitely deep profile typical for many applications such as water fluxes for both infiltration and evaporation between ground surface and saturated zone. We addressed the significance of ground surface conditions, structural arrangement within the unit cell, and the number of repeated units on the applicability of harmonic mean of hydraulic conductivities in representing the heterogeneous layered medium. Figure 7 showed flux prediction and its associated relative error, and capillary pressure profile for binary heterogeneous structure. It was demonstrated that structural arrangement of the repeated unit is significant. Specifically, the location of coarse material in the unit cell is an important indicator of whether using harmonic mean overpredicts or underpredicts the flux of the structured layered heterogeneous medium. Results indicated that using harmonic mean of individual hydraulic conductivities is appropriate only when the number of repeated structured layers is larger than about 100. It was also found that the harmonic mean of hydraulic 
conductivities performs better for infiltration than evaporation in representing fluxes of the layered heterogeneous soils.
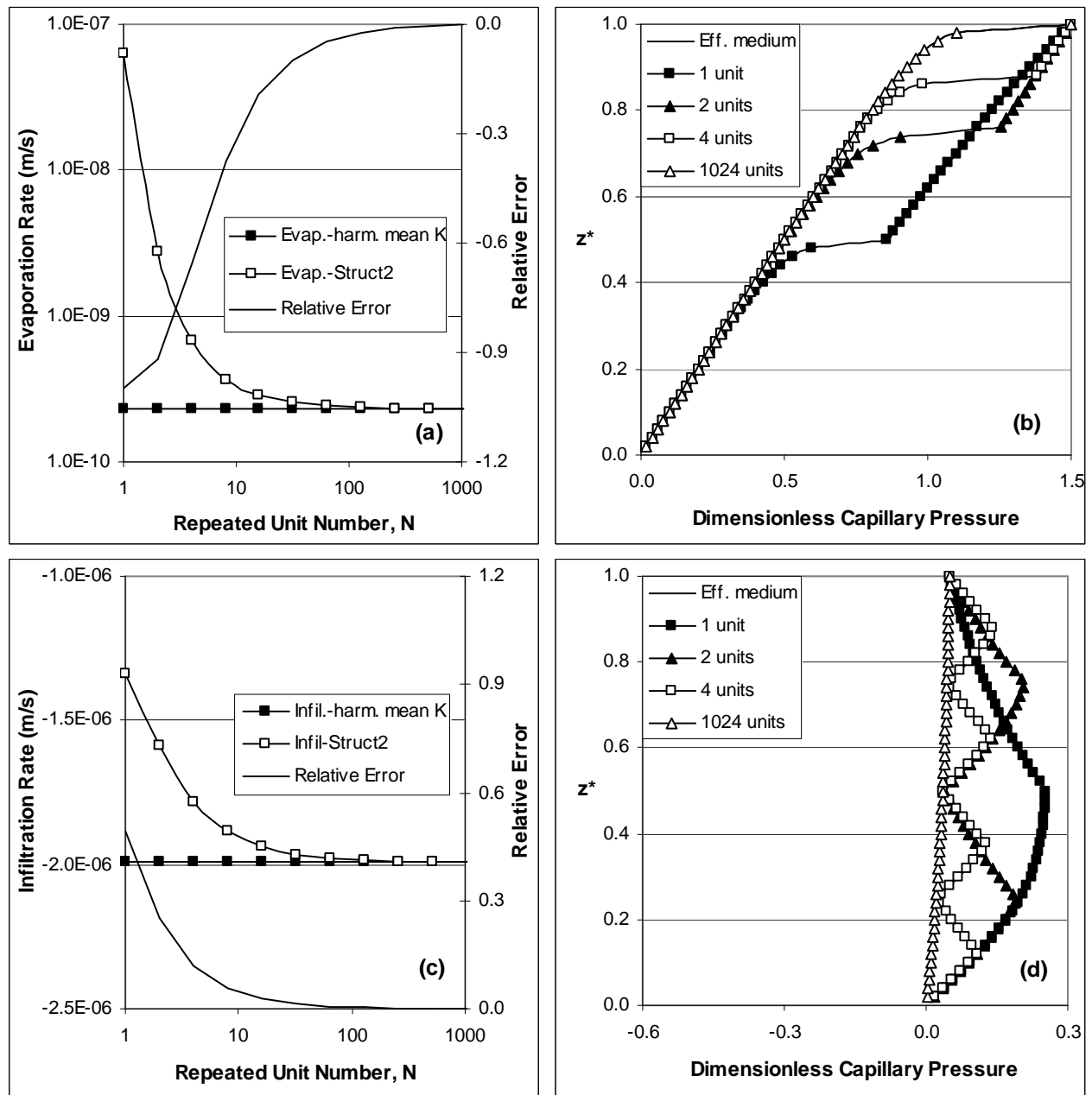

Figure 7: Flux prediction and its associated relative error, and capillary pressure profile for binary structure with $\langle K s\rangle=1.78 \times 10^{-5} \mathrm{~ms}^{-1}$ and $\left\langle\alpha^{*}>=7.0\right.$. (a) Flux and associated relative error, $h=$ 1.5 (evaporation), (b) Capillary pressure profile, $h=1.5$ (evaporation), (c) Flux and associated relative error, $h=0.05$ (infiltration), and (d) Capillary pressure profile, $h=0.05$ (infiltration)

\section{Incorporating Soil Hydraulic Parameter Statistics into Developing Neural Network Based Pedo-transfer Functions}

The traditional ANN based PTFs, in general, is to adjust ANN's coefficients to solely minimize the difference between the estimated and measured soil hydraulic parameters in the neural network training process. The training process, however, does not consider the distributions of soil hydraulic parameters and the trained neural networks may yield improper distributions and unrealistic correlations between the output parameters, which may severely affect probabilistic predictions. In the study of Gautam et al. (2010), we incorporated the distributions and correlations of the soil hydraulic parameters into the ANN PTF development by adding two regularization terms to the ANN error functions. The traditional neural network based PTFs utilize the objective function (denoted MSE $E F$ ) (i.e., the sum of the squared errors of the neural network prediction of the soil 
hydraulic parameters or mean square error (MSE)). To incorporate the hydraulic parameter distributions, the error function (denoted $E F 2$ ) was modified to include hydraulic parameter means and variances. To resolve the problem of unrealistic parameter correlations introduced by the neural networks, an error function (denoted $E F 3$ ) was introduced to also include the parameter correlations among different hydraulic parameters.

$$
\begin{aligned}
& \text { MSE EF }=\sum_{i=1}^{N s} \sum_{j=1}^{N o} \alpha_{j}\left[\hat{Y}_{i j}-Y_{i j}\right]^{2} \\
& E F 2=\sum_{i=1}^{N s} \sum_{j=1}^{N o} \alpha_{j}\left[\hat{Y}_{i j}-Y_{i j}\right]^{2}+\sum_{j=1}^{N o} \gamma_{j}\left[\hat{\mu}_{j}-\mu_{j}\right]^{2}+\sum_{j=1}^{N o} \delta_{j}\left[\hat{\sigma}_{j}^{2}-\sigma_{j}^{2}\right]^{2} \\
& E F 3=\sum_{i=1}^{N s} \sum_{j=1}^{N o} \alpha_{j}\left[\hat{Y}_{i j}-Y_{i j}\right]^{2}+\sum_{j=1}^{N o} \gamma_{j}\left[\hat{\mu}_{j}-\mu_{j}\right]^{2}+\sum_{j=1}^{N o} \delta_{j}\left[\hat{\sigma}_{j}^{2}-\sigma_{j}^{2}\right]^{2}+\sum_{i=1}^{N o} \sum_{j=i+1}^{N o} \varepsilon_{j}\left(\widehat{C}_{i j}-C_{i j}\right)^{2}
\end{aligned}
$$

where $N_{s}$ is the number of samples, $N_{o}$ is the number of output parameters, $Y_{i j}$ is the measured hydraulic parameters and $\hat{Y}_{i j}$ is the predicted hydraulic parameters (i.e., $\theta_{r}, \theta_{s}$, $\alpha, n$, or $\left.K_{s}\right), \alpha_{j}$ are weighting coefficients that can be assigned based on measurement error and confidence level of $Y_{i j}$ and will not be adjusted in the neural network training process, $\gamma_{j}, \delta_{j}$ are weighting coefficients for the mean and variance, $\hat{\mu}_{j}$ and $\hat{\sigma}_{j}^{2}$ are the mean and variance of $\hat{Y}_{j}$ estimated by the neural networks, $\mu_{j}$ and $\sigma_{j}^{2}$ are the mean and variance of soil hydraulic parameter $Y_{j}$ assumed to be known a priori, $\varepsilon_{j}$ are pre-assigned weighting coefficients, $\hat{C}_{i j}$ and $C_{i j}$ are the correlations of the neural network estimated and measured parameters.

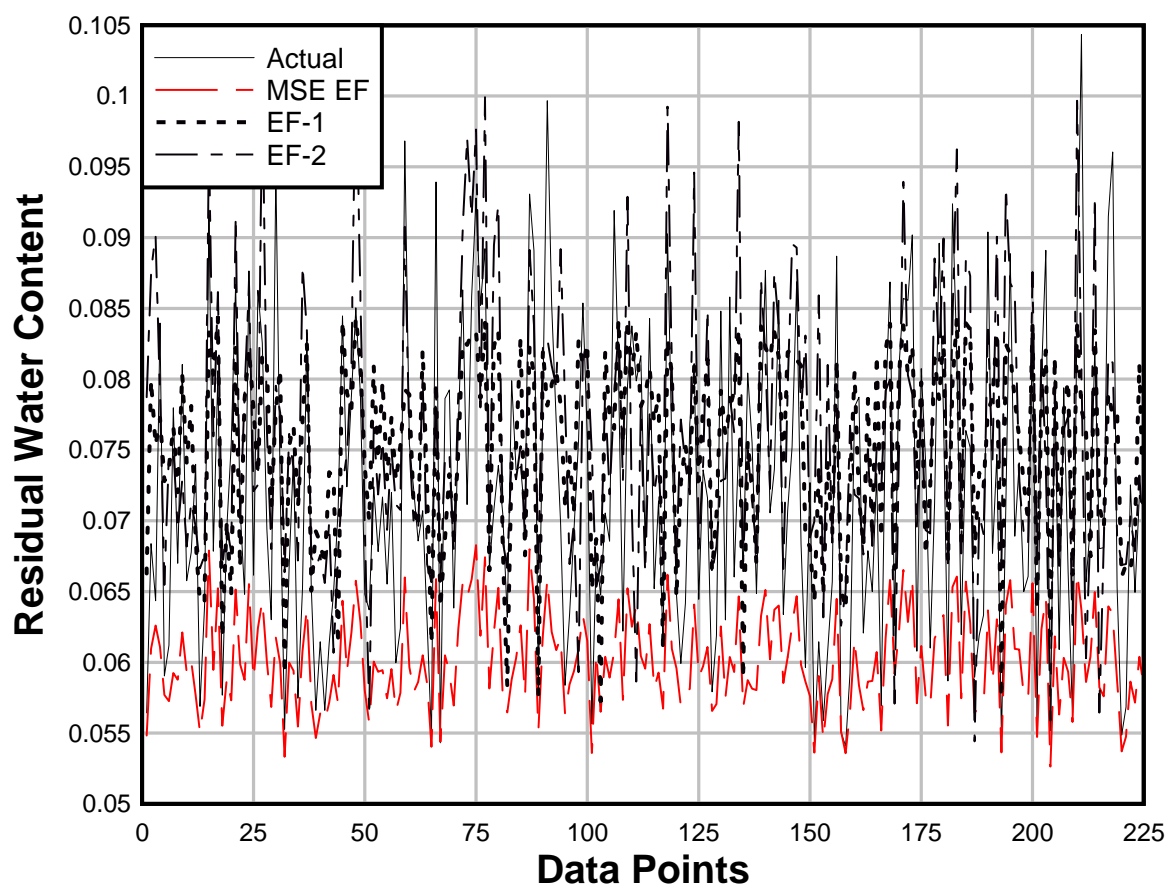

Figure 8: Comparison of actual and predicted residual water content in test phase 
We developed a suite of new neural network models to estimate soil hydraulic parameters. These neural networks have the same input and output variables, but different error functions, which incorporated sequentially the site soil hydraulic parameter measurements, parameter probability distributions, and parameter correlations. In order to illustrate the proposed approach, we used datasets using synthetic cases of different data availability. We employed a statistical multiple regression equation from the literature to generate synthetic data for this purpose. Figure 8 showed the comparison of actual and predicted residual water content in test phase. From the results with synthetic dataset and the Handford site dataset, we found that the modified ANN targeted for bias (mean) correction works quite well. While the modified ANN targeted for both bias and variance correction also works well, the correction in output variances is slight.

\section{Tension-Dependent Hydraulic Conductivity Anisotropy of Unsaturated Soils}

As mentioned earlier, one of key properties of the soil hydraulic parameters at the Hanford site is that there exists a layered structure. The layered structure causes anisotropy in unsaturated flow and contaminant transport. The effects of saturation degree (or tension) on hydraulic conductivity anisotropy in unsaturated soils have been recognized for long time, but they have not been fully described conceptually. Previous models included quantifying saturation-dependent anisotropy of soil formations that consist of many thin layers each with its own hydraulic properties characterized by a uniform density distribution of saturated hydraulic conductivity or soil bulk density. Some other approaches have also been developed to study the soil anisotropy behavior in dealing with flow and transport problems in saturated and unsaturated soils, such as tensorial connectivity-tortuosity concept.

In the study of Zhu and Sun (2010a), we investigated soil unsaturated hydraulic conductivity anisotropy that mainly arises from a combination of both wide range of soil texture variations and within narrow range of texture units in conjunction with pedotransfer functions (PTFs) of soil hydraulic properties. We developed a new approach to combine the neural network based PTF results with the thin layer approach to explore saturation-dependent anisotropy behavior for a wide range of texture and bulk density conditions. Anisotropy models were developed that quantify saturated and unsaturated hydraulic conductivities for soils composed of many thin layers distinguished by texture and bulk density. Figure 9 showed Relationships between $K h$ (hydraulic conductivity parallel to the layering), $K v$ (hydraulic conductivity perpendicular to the layering), and the anisotropy factor $A$, and the capillary pressure head $\psi$. Results indicated that the vertical variability of soil texture and bulk density was a significant factor that impacts tension-dependent hydraulic conductivity anisotropy of unsaturated soils. The coupled dependence of the hydraulic parameters on the texture and bulk density was important to determine the anisotropic behaviour of unsaturated soils. The minimum anisotropy at a certain capillary pressure was only observed when both $K_{S}$ and $\alpha$ were related to the soil particle diameter. When only one hydraulic parameter was related to the grain diameter or when both were not related to the same attribute simultaneously, the unsaturated soil anisotropy increased monotonically with the increasing capillary pressure head. The inter-relationships of soil texture, bulk density, and hydraulic properties may cause vastly different anisotropy behaviours of the unsaturated soils. The correlation between the soil 
grain diameter and bulk density decreased the anisotropy effects of the unsaturated layered soils.

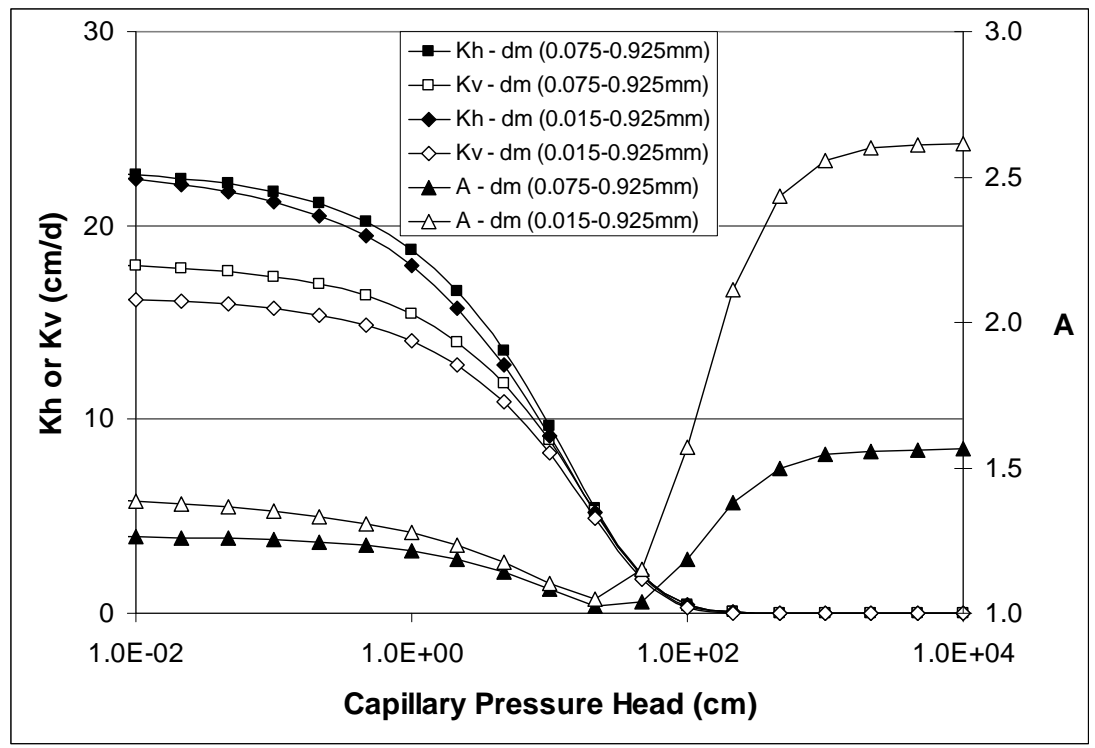

Figure 9: Relationships between $K h$ (hydraulic conductivity parallel to the layering), $K v$ (hydraulic conductivity perpendicular to the layering), and the anisotropy factor $A$, and the capillary pressure head $\psi$. van Genuchten $n=1.59$, and the correlation coefficient between $\ln d m$ and $\ln \rho, r=0.8$.

Scenario 1: Both $K_{S}$ and $\alpha$ are empirically related to $d m$ through linear regressions
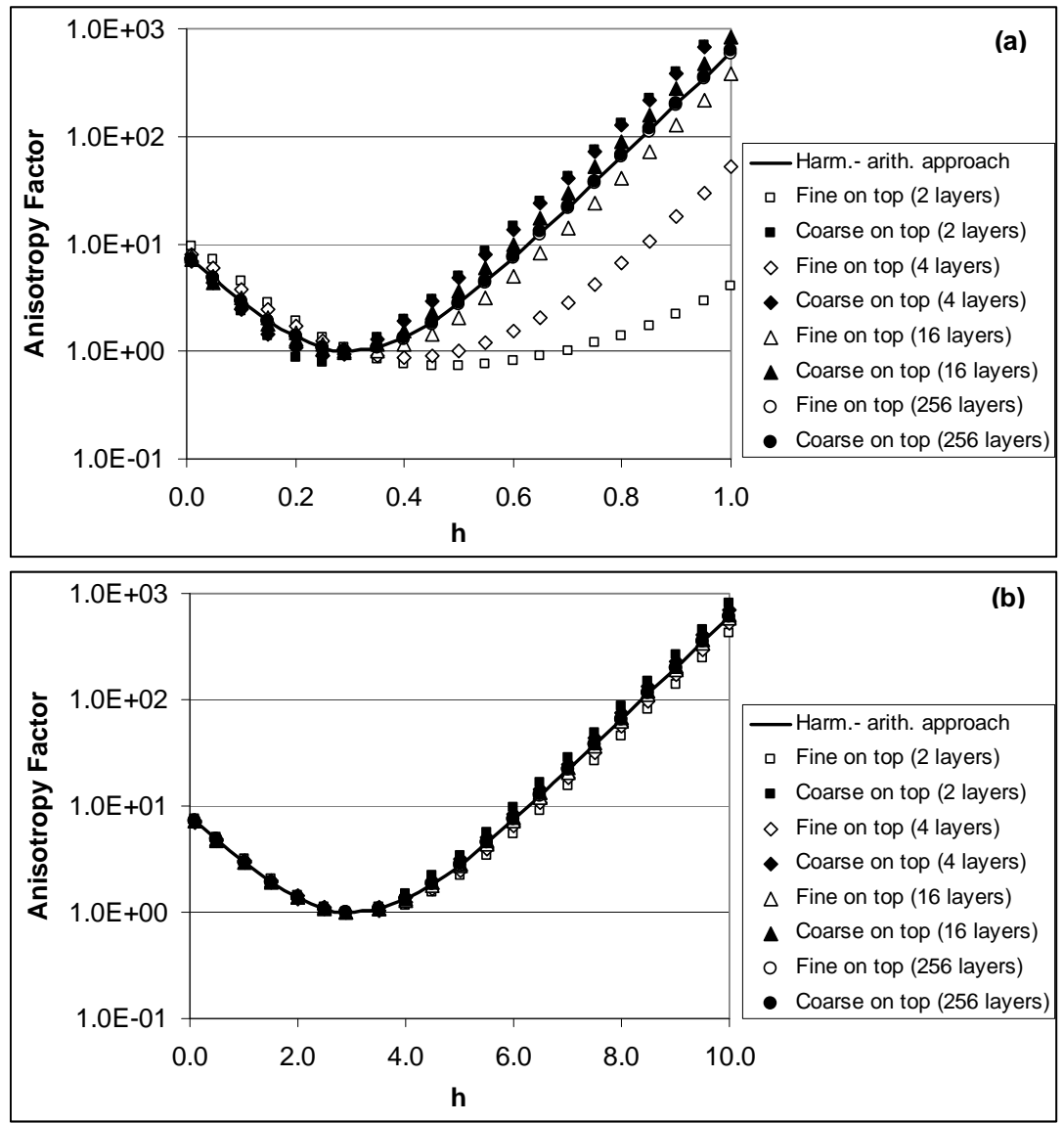

Figure 10: Anisotropy factor for (a) $1 \mathrm{~m}$ profile, and (b) $0.1 \mathrm{~m}$ profile 
The main objective of the study by Zhu (2010) was to improve basic understanding of capillary pressure-dependent soil hydraulic conductivity anisotropy by examining how the anisotropy characteristics are related to the layered structure of soils. Specifically, we investigated the hydraulic conductivity anisotropy of layered soils bound by a same capillary pressure at both ends of the domain. Both randomly arranged layered soils and structurally heterogeneous soils of repeated unit cells of homogeneous sublayers were considered. The anisotropy was determined by the fluxes in vertical and horizontal directions subject to the same capillary pressure. The impact of various conditions was examined and discussed. Figure 10 showed anisotropy factor for both thick and thin soil profiles. Results demonstrated that many factors such as capillary pressure, number and structure of the layers and correlations of layer hydraulic parameters significantly impact the anisotropy of unsaturated soils.

\section{Soil Hydraulic Properties for Moisture Redistribution in Large Scale Heterogeneous Soils}

The study of Zhu and Sun (2010b) focused on developing effective soil hydraulic parameters that can be used to predict average near surface moisture evolution of large scale heterogeneous fields. The main objective of this study was to develop guidelines of how to develop effective hydraulic parameters capable of simulating average infiltration and subsequent moisture redistribution over a large scale heterogeneous field. The heterogeneous soil was represented by a series of vertically homogeneous stream tubes or parallel columns. Average large-scale infiltration and redistribution in the heterogeneous soils was quantified through Monte Carlo simulations of multiple realizations (stream tubes) of local-scale infiltration and subsequent soil moisture redistribution. The effective parameters were sought to mimic the total average amount of infiltrated water into the soil, and to capture the subsequent surface soil moisture redistribution averaged over the large heterogeneous soils. Simple hydraulic parameter aggregation schemes were developed where effective parameter was derived only for one hydraulic parameter while keeping the other parameters at their arithmetic means. The derived schemes while simple were capable of simulating large scale infiltration and subsequent redistribution behavior. Results demonstrated that effective hydraulic parameters typically exhibit a step change from infiltration to redistribution with the size of the step change being related to the degree of hydraulic parameter heterogeneity and the correlations among the hydraulic parameters. However, the effective parameters do not change significantly over time for soil moisture redistribution. This finding is particularly useful in practical applications when simulating large scale soil moisture redistribution in that relatively constant effective hydraulic parameters are adequate for predicting transient behavior of soil moisture redistribution.

Average surface water content evolution is mainly determined by mean hydraulic parameters. When only the saturated hydraulic conductivity is heterogeneous, the effective parameter does not change significantly and is between arithmetic mean and geometric mean. When only the saturated water content is heterogeneous, the effective parameter is between geometric mean and arithmetic mean for initial absorption and is above arithmetic mean for subsequent redistribution. When only the shape parameter is variable, the effective parameter is between geometric mean and harmonic mean for initial absorption and is below harmonic mean for subsequent redistribution. 

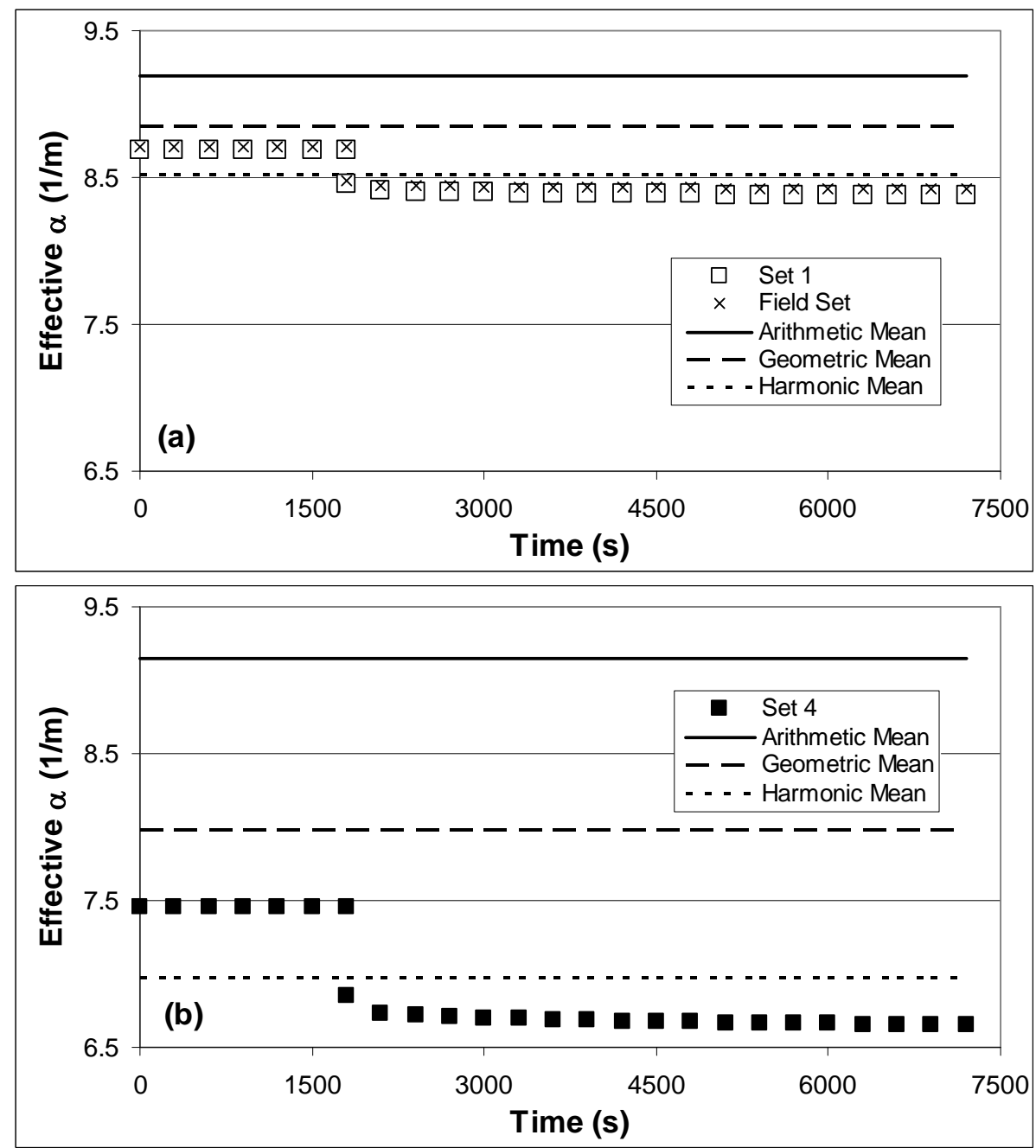

Figure 11: Effective $\alpha$ for infiltration (absorption) and subsequent redistribution. Only one hydraulic parameter $\left(\theta_{\mathrm{s}}, \mathrm{K}_{\mathrm{s}}\right.$ or $\left.\alpha\right)$ was variable, and others were kept at arithmetic mean when calculating average infiltration and redistribution

For a more realistic situation when all the three hydraulic parameters are variable, the effective parameter ranges from below harmonic mean to above arithmetic mean. Correlations among hydraulic parameters have more significant impact on effective parameters for initial absorption and have relatively insignificant effect for subsequent redistribution when all three hydraulic parameters are variable. 


\section{LIST OF PAPERS AND PRESENTATIONS/ABSTRACTS}

\section{A: Peer Reviewed Journal Papers/Manuscripts}

Deng, H., M. Ye, M. G. Schaap, and R. Khaleel. 2009. Quantification of uncertainty in pedotransfer function-based parameter estimation for unsaturated flow modeling. Water Resources Research 45:W04409, doi:10.1029/2008WR007477.

Gautam, M., J. Zhu, M. Ye, P. Meyer, and A. Hassan. 2010. Modified artificial neural network pedotransfer function of soil hydraulic properties. manuscript in preparation, to be submitted to European Journal of Soil Science.

Ye, M., M. G. Schaap, R. Khaleel, and J. Zhu. 2007. Simulation of field injection experiments in heterogeneous unsaturated media using cokriging and artificial neural network. Water Resources Research 43:W07413, doi:10.1029/2006WR005030.

Ye, M., and R. Khaleel. 2008. A Markov chain model for characterizing medium heterogeneity and sediment layering structure. Water Resources Research 44: W09427, doi:10.1029/2008WR006924.

Zhu, J. 2008, Equivalent parallel and perpendicular unsaturated hydraulic conductivities: Arithmetic mean or harmonic Mean?. Soil Science Society of America Journal 72: 1226-1233, doi:10.2136/sssaj2007.0337.

Zhu, J. 2010. Soil layer structure and capillary pressure dependent hydraulic conductivity anisotropy, manuscript in preparation, to be submitted to Soil Science.

Zhu, J., and D. Sun. 2009. Effective soil hydraulic parameters for transient flows in heterogeneous soils. Vadose Zone Journal 8:301-309, doi:10.2136/vzj2008.0004.

Zhu, J., and D. Sun. 2010a. Capillary pressure-dependent anisotropy of unsaturated soils. Canadian Journal of Soil Science, in press.

Zhu, J., and D. Sun. 2010b. Soil hydraulic properties for moisture redistribution in large scale heterogeneous landscape. Gerderma, to be submitted.

Zhu, J., and A. W. Warrick. 2009. Unsaturated hydraulic conductivity for layered soils of structured heterogeneity. Vadose Zone Journal, under revision.

B: Partial List of Presentations/Abstracts at Conferences Benefited from the Support

Zhu, J., and D. Sun, Soil hydraulic properties for large scale transient absorption and redistribution, EGU General Assembly 2010, May 2 - 7, 2010, Vienna, Austria. submitted.

Zhu, J., Saturation-dependent anisotropy of structured and random layered soils, EGU General Assembly 2010, May 2 - 7, 2010, Vienna, Austria. submitted.

Gautam, M., J. Zhu, M. Ye, P. Meyer, and A. Hassan, Modified ANN-pedotransfer function for soil hydraulic properties, W2188 USDA Multi-State Research Meeting, January 4 - 6, 2010, Las Vegas, Nevada, U. S. A.

Zhu, J., and D. Sun, Effective soil hydraulic properties for infiltration and redistribution in unsaturated zone, AGU Fall Meeting, December 14-18, 2009, San Francisco, California, U. S. A.

Zhu, J., Capillary pressure-dependent anisotropy of unsaturated layered soils, ASACSSA-SSSA 2009 International Annual Meetings, November 1-5, 2009, Pittsburgh, Pennsylvania, U. S. A. 
Sun, D., and J. Zhu, Soil hydraulic parameters for evaporation in large scale heterogeneous soils, ASA-CSSA-SSSA 2009 International Annual Meetings, November 1-5, 2009, Pittsburgh, Pennsylvania, U. S. A.

Sun, D., and J. Zhu, Averaging schemes of hydraulic properties in large scale heterogeneous soils, GSA Annual Meeting, October 18-21, 2009, Portland, Oregon, U. S. A.

Sun, D., and J. Zhu, Soil hydraulic properties and large scale evapotranspiration in desert ecosystem, 94th ESA Annual Meeting, August 2-7, 2009, Albuquerque, New Mexico, U. S. A.

Zhu, J., and D. Sun, Effect of spatial soil hydraulic properties on large scale evapotranspiration, AGU Joint Assembly, May 24-27, 2009, Toronto, Ontario, Canada.

Zhu, J., and A. W. Warrick, Effective hydraulic conductivity for layered soils of structured heterogeneity, W1188 USDA Multi-State Research Meeting, January 5 7, 2009, Oracle, Arizona, U. S. A.

Zhu, J., Unsaturated hydraulic conductivity for layered heterogeneous soils, AGU 2008 Fall Meeting, December 15-19, 2008, San Francisco, California, U. S. A.

Gautam, M., J. Zhu, M. Ye, P. D. Meyer, and A. E. Hassan, Improved artificial neural network-pedotransfer functions (ANN-PTFs) for estimating soil hydraulic parameters, AGU 2008 Fall Meeting, December 15-19, 2008, San Francisco, California, U. S. A.

Zhu, J., Macroscopic unsaturated hydraulic conductivities, 2008 GSA and ASA-CSSASSSA Joint Annual Meeting, 5-9 October 2008, Houston, Texas, U. S. A.

Sun, D., and J. Zhu, Effective soil hydraulic properties for heterogeneous fields, 2008 GSA and ASA-CSSA-SSSA Joint Annual Meeting, 5-9 October 2008, Houston, Texas, U. S. A.

Zhu, J., Effective soil hydraulic properties for heterogeneous soils: Effect of temporal scales, 2008 Symposium: Understanding Near-Surface Environmental Processes, Desert Research Institute and Nevada Water Resources Association, April 9 - 10, 2008, Las Vegas, Nevada, U. S. A.

Zhu, J., Saturation-dependent anisotropy of unsaturated soils, 2008 Symposium: Understanding Near-Surface Environmental Processes, Desert Research Institute and Nevada Water Resources Association, April 9 - 10, 2008, Las Vegas, Nevada, U. S. A.

Zhu, J., Equivalent unsaturated hydraulic conductivities for layered soils: Arithmetic mean or harmonic mean?, W1188 USDA Multi-State Research Meeting, January 2 4, 2008, Las Vegas, Nevada, U. S. A.

Zhu, J., and D. Sun, and S. Neelakrishnan, Hydraulic conductivity anisotropy of unsaturated soils, AGU 2007 Fall Meeting, December 10-14, 2007, San Francisco, California, U. S. A.

Zhao, Y., J. Zhu, M. Ye, P. D. Meyer, F. Pan, and A. E. Hassan, Incorporating soil hydraulic parameter statistics in developing pedo-transfer functions, AGU 2007 Fall Meeting, December 10-14, 2007, San Francisco, California, U. S. A.

Zhu, J., Parallel and perpendicular unsaturated hydraulic conductivities for layered soils, ASA-CSSA-SSSA 2007 International Annual Meetings, November 4-8, 2007, New Orleans, Louisiana, U. S. A. 
Sun, D., and J. Zhu, Effective hydraulic parameters for transient infiltration in heterogeneous soil, GSA 2007 Annual Meeting, October 28-31, 2007, Denver, Colorado, U. S. A.

Zhu, J., M. Ye, P. D. Meyer, F. Pan, and A. E. Hassan, Upscaling schemes of hydraulic properties and characterizing hydraulic parameter variability using cokriging and artificial neural network for heterogeneous soils, W1188 Multistate Research Project Annual Meeting, January 2 - 4, 2007, Las Vegas, Nevada, U. S. A.

Mohanty, B. P., A. V. M. Ines, J. Zhu, R. Jana, N. N. Das, and S. K. Sharma, Effective soil hydraulic parameters across scales for land-atmosphere interaction, AGU 2006 Fall Meeting, December 11-15, 2006, San Francisco, California, U. S. A.

Sun, D., J. Zhu, and M. H. Young, Effective hydraulic properties for large scale transient infiltration in heterogeneous formations, GSA 2006 Annual Meeting, October 22-25, 2006, Philadelphia, Pennsylvania, U. S. A.

Zhu, J., B. P. Mohanty, and N. N. Das, Effective soil hydraulic properties at the landscape scale and beyond, 18th World Congress of Soil Science, July 9-15, 2006, Philadelphia, Pennsylvania, U. S. A.

Zhu, J., D. Sun, and M. H. Young, Aggregating hydraulic property measurements to large scale hydrologic processes, Western Pacific Geophysics Meeting, July 24 - 27, 2006, Beijing, China.

Zhu, J., M. H. Young, and M. Th. van Genuchten, Upscaling schemes for hydraulic functions at the landscape scale, AGU Joint Assembly, May 23 - 26, 2006, Baltimore, Maryland, U. S. A.

Zhu, J., and M. H. Young, Upscaling relationships of hydraulic functions for flux and moisture in heterogeneous soils, W1188 Multistate Research Project Annual Meeting, January 2 - 4, 2006, Las Vegas, Nevada, U. S. A.

\section{LIST OF PEOPLE WORKING ON THE PROJECT}

Ms. Yanxia Zhao ( 50\%), a MS student at University of Nevada Las Vegas. She mainly worked on the development of artificial neural network to predict soil hydraulic properties by incorporating parameter distributions and correlations into the developed ANN pedo-transfer functions. But in September 2007, she withdrew from the MS program due to family reason.

Dr. Mahesh Gautam ( 30\%), postdoctoral fellow. Since graduate student Yanxia Zhao withdrew in 2007, Dr. Gautam was hired as a postdoctoral fellow mainly to work on new neural network model development from September 2008 to December 2009.

Mr. Shankar Neelakrishnan ( $10 \%)$, a MS student at University of Nevada Las Vegas. He worked as a full-time summer student during May - August 2007 (full time during the summer) to help in numerical analysis.

Mr. Rushikesh Veni ( 10\%), a MS. student at University of Nevada Las Vegas. He was hired as full-time summer student during May - August 2008 (full time during the summer) to help in numerical analysis. 
Mr. Feng Pan $(\sim 10 \%)$, a PhD student at University of Nevada Las Vegas. He helped in numerical analysis and random field generations.

Dr. Jianting Zhu ( 25\%), PI. He oversaw the project and mainly worked on field scale effective soil hydraulic properties and hydraulic conductivity anisotropy typical of layered soils at Hanford site.

Dr. Ming Ye $(\sim 10 \%)$, co-PI. He left for Florida State University in 2007 and was involved in the project. He mainly worked on geostatistical and uncertainty analysis of soil hydraulic parameters.

Dr. Ahmed Hassan $(<5 \%)$, co-PI. He was consulted with and assisted in the artificial neural network development.

Mr. Craig Shirley, co-PI. He left DRI only a few months after the grant started, so he did not work nor received financial support from this project. PI Zhu took over his responsibility.

Dr. Philip Meyer, the national laboratory collaborator with no financial support from this project. He was consulted on the soil hydraulic property data at the Hanford site, artificial neural network development and Bayesian updating etc.

\section{Other Collaborators}

Thank to the support from DOE EPSCoR program, the PI and co-PI were also able to initiate collaboration with additional researchers in soil hydraulic property heterogeneity and anisotropy studies. The following is a partial list of researchers who collaborated with PI and co-PI but did not receive direct financial support from this project: D. Sun (University of Houston, Clear Lake); M. G. Schaap (University of Arizona); R. Khaleel (Fluor Government Group); A. W. Warrick (University of Arizona); M. H. Young (Desert Research Institute); M. Th. van Genuchten (Universidade Federal do Rio de Janeiro, Brazil).

\section{UPDATE LIST OF OTHER SUPPORT}

\section{Project Title: FY06 Nevada Water Resources Research Institute Base Program} Sponsor: DOI-USGS

Total Amount: \$460,000, 03/01/06 -2/28/11

Time Commitment: 0.40 month/yr

This project is to develop hydraulic property correspondence and upscaling for arid and semi-arid hydrologic processes and uncertainty and sensitivity of ground-water discharge estimates for the shrublands in the Great Basin area. It also deals with soil hydraulic properties, but mainly investigates the relationships among different hydraulic property models based on field-measured hydraulic parameters characterized by different hydraulic functions. This project has some overlap with and leverage over 
the DOE EPSCoR project on the task of effective hydraulic properties and anisotropy of layered heterogeneous soils.

2. Project Title: Analyzing Groundwater Fluctuations to Better Estimate Water Use through ET by Tamarisk

Sponsor: DOI-Bureau of Reclamation

Total Amount: \$167,503, 10/01/2008 - 03/01/2011

Time Commitment: 2 month/yr

This project is to perform uncertainty analysis of groundwater evapotranspiration by phreatophyte in the riparian zone of Colorado River. There is no overlap with the DOEEPSCoR project.

3. Project Title: Integration of Climate Change Impacts into Risk and Uncertainty Analyses

Sponsor: Army Corps of Engineers

Total Amount: \$120,000, 10/01/2008 - 07/31/2010

Time Commitment: 1.5 months/yr

This project is to investigate the potential future climate change on flood risks in arid and semi-arid regions of southwestern U. S. A. There is no overlap with the DOE-EPSCoR project.

4. Project Title: Significance of Groundwater Divide in Water Accounting and Return Flow Calculation for Lower Colorado River Aquifers

Sponsor: DOI-Bureau of Reclamation

Total Amount: \$264,387, 10/01/2009 - 01/01/2011

Time Commitment: 4 months/yr

This project is to delineate possible groundwater divide that will influence groundwater flow direction in the local irrigation districts along the Colorado River. There is no overlap with the DOE-EPSCoR project.

5. Project Title: Water Resources Evaluation Program - METRIC Uncertainty Analysis Sponsor: DOI-Bureau of Reclamation

Total Amount: \$245,184, 09/17/2009 - 09/30/2012

Time Commitment: 1.5 month/yr

This project is to estimate evapotranspiration water budget in Nevada using METRIC computer code. PI's responsibility is uncertainty and sensitivity analysis of ET estimates. There is a slight overlap with the DOE-EPSCoR project, given uncertainty and sensitivity is also part of the DOE-EPSCoR project. 